\title{
Bilateral Third Nerve Palsy in Mirror Aneurysms of the Posterior Communicating Arteries
}

Enrique Gomez-Figueroa, Omar Cardenas-Saenz, Gerardo Quiñones-Pesqueira, Roberto Cervantes-Uribe, Juan Manuel Calleja-Castillo

Vascular Neurology Department, Instituto Nacional de Neurologia y Neurocirugia, Mexico City, Mexico

Received: 05/05/2018

Accepted: 09/06/2018

Published: 28/06/2018

How to cite this article: Gomez-Figueroa E, Cardenas-Saenz O, Quiñones-Pesqueira G, Cervantes-Uribe R, Calleja-Castillo JM. Bilateral third nerve palsæ in mirror aneurysm of the posterior communicating arteries. EJCRIM 2018;5: doi:10.12890/2018_000912.

Contributions: Dr. Calleja-Castillo was in charge of the diagnosis, care and follow-up of the patient and supervised the writing of the manuscript.

Dr. Gomez-Figueroa prepared the draft. All authors were part of the patient care team and approved the final version of the manuscript.

Conflicts of Interests: The Authors declare that there are no competing interests.

This article is licensed under a Commons Attribution Non-Commercial 4.0 License

\section{ABSTRACT}

Background: Bilateral third cranial nerve palsy has only been reported in a handful of conditions including some with inflammatory, tumoural and vascular causes. An urgent imaging study is mandatory to rule out vascular aetiology, mainly aneurysmal subarachnoid haemorrhage (aSAH).

Case presentation: A 28-year-old Hispanic woman presented to the emergency department with a 21-day history of a sudden-onset and severe headache that on three occasions was accompanied by transient loss of awareness, the last episode occurring a week previously. The simple CT image showed minimal bleeding at the level of the perimesencephalic cisterns, with evidence of SAH. An angioCT revealed a $5 \times 6 \mathrm{~mm}$ bilobed saccular aneurysm of the right posterior communicating artery and a $2 \times 2 \mathrm{~mm}$ saccular aneurysm in the posterior left communicating artery.

Conclusions: A mirror aneurysm is found in 2-25\% of aSAH cases. To date there is no consensus about the optimal management of patients with these findings.

\section{LEARNING POINTS}

- The presence of third cranial nerve palsy should always raise the suspicion of an aneurysm.

- Subarachnoid haemorrhage is the most common cause of a thunderclap headache.

- Aneurysmal subarachnoid haemorrhage requires surgical management in all cases.

\section{KEYWORDS}

Third nerve palsy, oculomotor nerve palsy, subarachnoid hemorrhage, mirror aneurysm, thunderclap headache

\section{CASE PRESENTATION}

A 28-year-old Hispanic woman presented to the emergency department with a 21-day history of a sudden-onset and severe headache that on three occasions was accompanied by transient loss of awareness, the last episode occurring a week previously. She had no other relevant medical history.

The general examination at admission was normal but on the neurological examination there was evidence of mydriasis and bilateral third cranial nerve palsy (Fig. 1) 


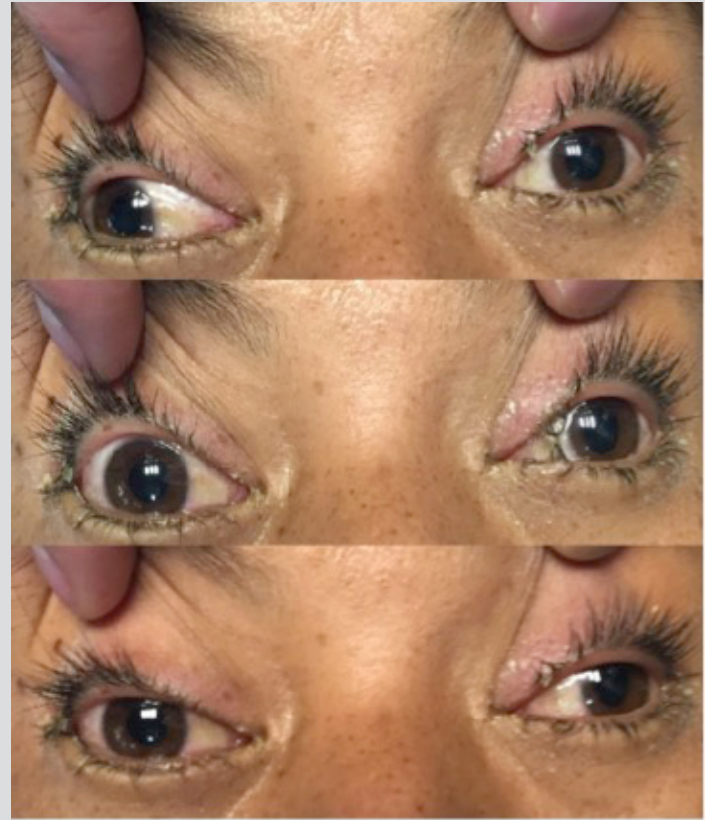

Figure 1. Bilateral oculomotor palsy. Primary gaze in the patient (middle panel). Evaluation of horizontal eye movements in the patient to the right (top panel) and to the left (bottom panel). Bilateral mydriasis is also seen

The simple CT image showed minimal bleeding at the level of the perimesencephalic cisterns, with evidence of subarachnoid haemorrhage (SAH). An angioCT was performed and revealed a $5 \times 6 \mathrm{~mm}$ bilobed saccular aneurysm of the right posterior communicating artery and a $2 \times 2 \mathrm{~mm}$ saccular aneurysm in the posterior left communicating artery (Fig. 2). The patient underwent surgical clipping of both aneurysms without complications.

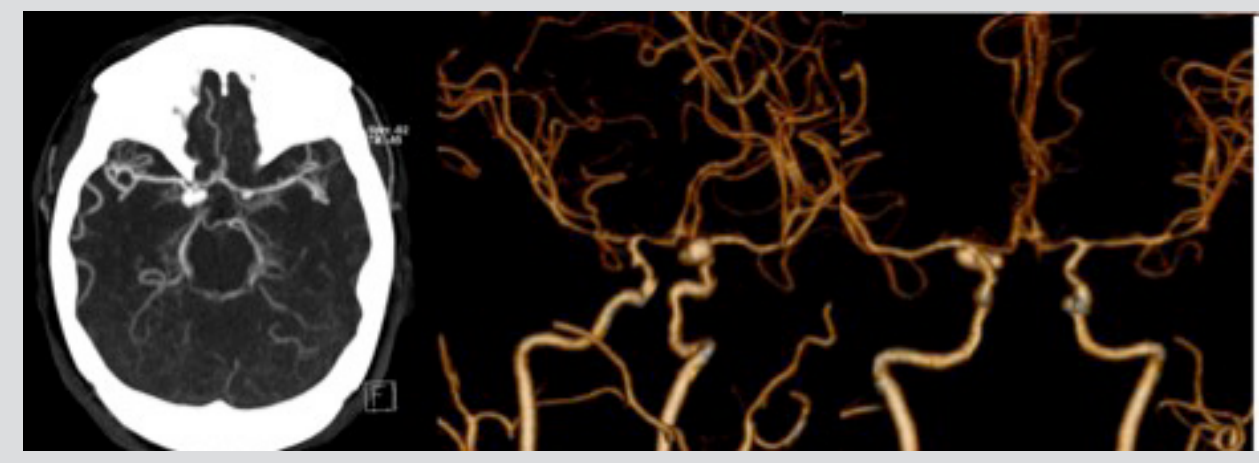

Figure 2. Computed tomography angiography revealing the mirror aneurysms

\section{DISCUSSION}

Initial assessment of third cranial nerve palsy involves checking for pupil involvement and other neurological symptoms suggestive of the site of involvement. Anatomically, two sites can be considered for the isolated lesion: the mesencephalon and the subarachnoid space ${ }^{[1]}$. Previously reported causes of bilateral involvement of the oculomotor nerve include vasculitis, diabetes mellitus, infections (meningovascular syphilis, Lyme disease or mucormycosis), tumours, polyneuropathy (Guillain-Barre syndrome, Miller-Fisher syndrome or acute dysautonomia), oculomotor pseudoparalysis (myasthenia gravis, Graves ophthalmopathy or chronic progressive ophthalmoplegia), intracranial hypotension and $\mathrm{SAH}^{[2]}$.

Patients with an acute acquired third nerve palsy usually complain of sudden onset of binocular horizontal, vertical or oblique diplopia and a droopy eyelid. Infrequently, the patient is aware of an enlarged pupil. Sudden, severe ('the worst headache of my life') headache (thunderclap) might suggest SAH due to a ruptured aneurysm as the cause of the third nerve palsy, as in this case. Aneurysmal compression 
of the superficial pupil fibres of the third nerve in the subarachnoid space results in a dilated and unresponsive pupil.

Imaging studies used to diagnose or exclude an intracranial aneurysm include contrast-enhanced MRI with MR angiography (MRA), computed tomographic angiography (CTA) and cerebral angiography. Cerebral angiography remains the gold standard but is invasive and associated with infrequent but significant risk $^{[3]}$.

The presence of headache with thunderclap characteristics in our patient and bilateral pupillary involvement raised the suspicion of a basilar aneurysmal lesion, although the actual findings were posterior communicating artery mirror aneurysms.

Mirror intracranial aneurysms occur symmetrically at the corresponding intracranial arteries. These aneurysms represent less than $5 \%$ of all intracranial aneurysms, but account for as much as $40 \%$ of all multiple intracranial aneurysms. The usual location is in the middle cerebral, pericallosal or internal carotid arteries ${ }^{[4]}$.

Only a few studies have examined the genetic predisposition to mirror aneurysms and other risk factors such as age, sex, hypertension, alcohol consumption and smoking. Patients with mirror aneurysms were more likely to be women and to report a family history of aneurysmal $\mathrm{SAH}^{[5,6]}$

One theory suggests that mirror aneurysms are due to a congenital predisposition that causes alterations in the blood flow during embryogenesis and that can help to differentiate this type of aneurysm from others. It is known that women are more susceptible to having these kinds of aneurysms and that a positive family history makes them more susceptible to mirror aneurysms. As mentioned above, there are also other risk factors that are modifiable, such as alcohol and tobacco consumption, which are risk factors for any type of aneurysm ${ }^{[6]}$. One of the studies performed by the National Institutes of Health $(\mathrm{NIH})$ and International Study of Unruptured Intracranial Aneurysms (ISUIA) investigators ${ }^{[6]}$ concluded that mirror aneurysms do not seem to predict future SAH, increased mortality or worsening of neurological status after 1 and 5 years of follow-up. These findings are important for making decisions about when to treat patients with mirror aneurysms; however, special attention should be given to recognized risk factors for rupture, including the size and location of the aneurysms, the presence of a high AR (aspect ratio, depth/neck width) and a high SR (size ratio, depth/parent vessel diameter), patient age and the presence of previous $\mathrm{SAH}$, as was the case in our patient ${ }^{[6-9]}$.

\section{REFERENCES}

1. Brazis P. Localization of lesions of the oculomotor nerve: recent concepts. Mayo Clin Proc 1991;66:1029-1035.

2. Lazaridis C, Torabi A, Cannon S. Bilateral third nerve palsy and temporal arteritis. Arch Neurol 2005;62:1766-1768.

3. Schwedt TJ, Matharu MS, Dodick DW. Thunderclap headache. Lancet Neurol 2006;5:621-631.

4. Roethlisberger M, Achermann R, Bawarjan S, Stienen MN, Fung C, D'Alonzo D, et al. Predictors of occurrence and anatomic distribution of multiple aneurysms in patients with aneurysmal subarachnoid hemorrhage. World Neurosurg 2018;111:e199-e205.

5. Schuss P, Konczalla J, Platz J, et al. Aneurysm-related subarachnoid hemorrhage and acute subdural hematoma: single-center series and systematic review. J Neurosurg 2013;118:984.

6. Meissner I, Torner J, Huston J, Rajput ML, Wiebers DO, Jones LK, Brown Jr RD, et al. Mirror aneurysms: a reflection on natural history. J Neurosurg 2012;116:1238-1241.

7. Wang R, Zhang D, Zhao J, Wang S, Zhao Y, Niu H. A comparative study of 43 patients with mirror-like intracranial aneurysms: risk factors, treatment, and prognosis. Neuropsychiatr Dis Treat 2014;10:2231-2237.

8. Doddasomayajula R, Chung BJ, Mut F, Jimenez CM, Hamzei-Sichani F, Putman CM, Cebral JR. Hemodynamic characteristics of ruptured and unruptured multiple aneurysms at mirror and ipsilateral locations. Am J Neuroradiol 2017;38:2301-2307.

9. Wang G, Liu J, Chen Y, et al. Morphological characteristics associated with the rupture risk of mirror posterior communicating artery aneurysms. J Neurolntervent Surg Published Online First: 02 February 2018. 